\title{
Probable risk factors for postoperative delirium in patients undergoing spinal surgery
}

\author{
Rui Gao $\cdot$ Zhi-zhou Yang $\cdot$ Ming Li $\cdot$ \\ Zhi-cai Shi $\cdot$ Qiang Fu
}

Received: 4 March 2008/Revised: 30 July 2008/ Accepted: 28 August 2008/Published online: 16 September 2008

(C) Springer-Verlag 2008

\begin{abstract}
Postoperative delirium and its risk factors had been widely reported in several kinds of surgeries; however, there is only one known article relative to postoperative delirium in spinal surgery. We retrospectively examined the incidence of postoperative delirium and the probable risk factors in patients undergoing spinal surgery in our hospital, with the same aged non-delirium patients as controls, over a 6-month period. Studies about postoperative delirium were reviewed and referenced for variable factors collecting in our study. $T$ tests, $\chi^{2}$ test and logistic regression analysis were performed to evaluate the various factors related to postoperative delirium. A total of 18 patients $(3.3 \%)$, all of them were aged 54 years or older, had postoperative delirium after surgery. Patients without postoperative delirium aged 54 years or older served as the control group. The percentage of patients older than 65 years $(P=0.003)$, with comorbid diseases such as diabetes mellitus $(P=0.042)$ or central nervous system disorders $(P=0.013)$, with a surgical history $(P=0.028)$ in delirium group was larger than the control group. The absolute number of medications being taken before the operation in the delirium patients was also more than the control group $(P=0.000)$. The percentage of patients transfused with $800 \mathrm{~mL}$ or more blood was also larger $(P=0.024)$ in delirium group was larger than the control group. Logistic regression analysis showed that central nervous system disorder (OR 6.480), surgical history (OR 3.499 ), age older than 65 years (OR 3.390), diabetes mellitus (OR 2.981), transfused $800 \mathrm{~mL}$ or more blood (OR
\end{abstract}

R. Gao $\cdot$ Z. Yang $\cdot$ M. Li · Z. Shi $\cdot$ Q. Fu $(\bowtie)$ Department of Orthopedics, Changhai Hospital, Second Military Medical University, 168 Changhai Road, 200433 Shanghai, China e-mail: RuiGao1984@hotmail.com; Johson.f@163.com
2.537), and hemoglobin less than $100 \mathrm{~g} / \mathrm{L}$ (OR 0.281) were significantly related to the occurrence postoperative delirium. Our findings suggest that postoperative delirium in spinal surgery can also occurred in younger patients and with an acceptable incidence in total. The risk for postoperative delirium is multifactorial. More prospective research is necessary in order to evaluate these and other risk factors in greater detail.

Keywords Postoperative delirium - Spinal surgery · Delirium observation screening (DOS) scale .

Logistic regression analysis

\section{Introduction}

It has been previously reported that postoperative delirium can occur following several kinds of surgery, including general surgery [17], head and neck cancer surgery [23], cardiac surgery [5, 13], vascular surgery [19], hip fracture [21, 24], joint replacement [22], and spinal surgery [12]. Postoperative delirium is defined as an acute state of confusion characterized by fluctuating consciousness and inattention, which in most studies occurs shortly after surgery. Diagnosis of this delirious state is usually done according to the confusion assessment method (CAM) criteria, the telephone interview for cognitive status (TICS, a modification of the Mini-Mental Status Exam [MMSE]), specific activity scale (SAS), administration of the delirium rating scale (DRS) or the NEECHAM confusion scale (NCS) [5, 12, 13, 19, 21, 23, 24]. Postoperative delirium has been related to many risk factors, including preoperative factors such as patient's age, drug use, sensory impairment, and the comorbid medical conditions; intraoperative factors such as type of surgery, type of 
anesthesia, duration of operation, blood loss, and amount of blood transfused; and postoperative factors such as laboratory data, blood pressure, and opiate analgesic drug use $[5,9,12,13,19,21,23,24]$. Patients who undergo surgery with these risk factors are considered to be prone to delirium after the operation. The incidence of postoperative delirium in general surgery, hip fracture, joint replacement, and open-heart surgery has been widely reported; however, there is only one known article that describes postoperative delirium in spinal surgery [12]. Delirious patients are often hospitalized longer than other patients and have higher morbidity and mortality rates [4, 14]. Prevention of delirium may help to reduce these problems and the associated costs, and thus there is a need for further understanding of the risk factors. This study was conducted retrospectively to examine the incidence and determine the probable risk factors of postoperative delirium in patients receiving spinal surgery.

\section{Patients and methods}

The subjects were 549 patients (302 males and 247 females) who underwent spinal surgery in Changhai Hospital, the Second Military Medical University between 1 May 2007 and 10 November 2007. Their age ranged from 10 to 83 years, with a mean of 48.2 years. Only one patient had a history of delirium prior to the operation. Patients without contraindications of sedatives had the option to receive oral diazepam ( 5 or $10 \mathrm{mg}$ ) on the day prior to the operation to relieve nervousness and anxiety, or to ensure high-quality sleep. All patients underwent the operation under general anesthesia. Phenobarbital (100 mg) and atropine $(0.5 \mathrm{mg})$ via intramuscular injection was given 0.5-1 h before surgery. Anesthetic agents, including propofol and isoflurance, or enflurance complex with fentanyl, rocuronium bromide or vecuronium bromide, were used during the operation. Patients that had a risk for abnormal vital signs were sent to the intensive care unit for at least $12 \mathrm{~h}$ after the operation. An adjustable and portable patient or patient-relative controlled analgesic pump (fentanyl) was used on most patients for postoperative pain relief.

Inouye summarized the clinical features of delirium: (1) acute onset, (2) fluctuating course, (3) inattention, (4) disorganized thinking, (5) altered level of consciousness, (6) perceptual disturbances, (7) psychomotor disturbances, (8) altered sleep-wake cycle, and (9) emotional disturbances [9]. Patients with the first and second features, and any two of the other features, were evaluated by nurses. Observations of subjects was conducted each shift and recorded using the delirium observation screening (DOS) scale. This scale was developed based on the DSM-IV criteria for delirium (American Psychiatric Association
1994) [3], literature review, clinical experience, and expert opinion. The DOS scale items can be observed by nurses during the regular care of the patients, and completing the scale takes only a few minutes. The scale shows high internal consistency (Cronbach's alpha, 0.96) and good predictive validity against a DSM-IV diagnosis $(P<0.001)$ [20]. The gold standard in this study was a DSM-IV diagnosis of delirium made by two senior spinal surgeons (Qiang Fu, Ming Li) and one psychiatrist (Yan Han). Each diagnosis was discussed by these three doctors.

Studies about postoperative delirium were reviewed and referenced for variable factors [5, 9, 12, 13, 17, 19, 21-24]. Preoperative factors included age, solitary life condition, drug treatment, preoperative hospitalized days, nutrition status, sensory impairment, comorbid diseases, surgical history, history of delirium, systolic blood pressure (SBP), hemoglobin (HGB), hematocrit (HCT), and blood biochemistry indexes (sodium, potassium, blood sugar). Alcohol and drug use were considered as one measure, with alcohol no less than $30 \mathrm{~g}$ per day and drug use as more than three kinds of drugs per day before operation. Hospitalization time in excess of 10 days was considered as a long hospital stay. The malnutrition diagnostic criteria were total protein less than $61 \mathrm{~g} / \mathrm{L}$. Patients with sensory impairment were those who had diagnosed glaucoma, cataracts, blindness or deafness. Comorbid diseases were hypertension, diabetes mellitus, pulmonary disease, cardiac disease, central nervous system disorder, psychiatric disorder, chronic kidney disease, and chronic liver disease.

The intraoperative factors included operative time, blood loss, blood transfusion, total infusion, and the use of surgical implants. Postoperative factors included the use of analgesic pumps, fever (temperature higher than $38^{\circ} \mathrm{C}$ ), SBP, HGB, HCT, and blood biochemistry index results of the first day after the operation. All the data was obtained from medical records or nursing records.

\section{Statistical analysis}

All data were analyzed by using SPSS 16.0. Numerical variables and ordinal variables were presented as mean and standard deviation (SD). Absolute numbers were for categorical variables. $T$ tests were used for statistical analysis of the difference in the mean values between the delirium group and non-delirium control group, and the $\chi^{2}$ test was used for the comparison of the categorical data. A $P$ value less than 0.05 was considered statistically significant. Logistic regression analysis was also used to evaluate the various factors related to postoperative delirium. The factors selected for analysis were those $P$ values close to or less than 0.2 in the previous univariate analysis, or those had significant clinical relevance. Stepwise forward 
method was used, and the entry and removal values were 0.10 and 0.15 , respectively.

\section{Results}

Postoperative delirium occurred in 18 of the 549 patients (3.3\%), 10 men and 8 women. The age of the patients with delirium ranged from 54 to 80 years, with a mean of 68.9 years. The delirium was observed between the day of the operation and 3 days after the operation, with a mean of 1.2 days. The duration of delirium ranged from 1 to 8 days, with a mean of 3.1 days. Of these patients, five patients had operation on the cervical spine, two on the cervico-thoracic spine, four on the lumbar-sacral spine, five on the lumbar spine and two on the thoraco-lumbar spine. Three patients required small amounts of perphenazine, between 2 and $4 \mathrm{mg} /$ day. Telephone follow-up interviews were performed every month from 57 days to 250 days. One patient died 36 days after operation in another hospital due to heart failure; this patient underwent an operation for traumatic dislocation of $\mathrm{C} 6$ and incomplete paralysis, with comorbid coronary heart disease and pulmonary emphysema. There were no patients requiring medical treatment, nor were there any patients with cognitive complications after discharge.

Since, none of the 549 patients younger than 54 years had delirium after the operation, patients aged 54 years or older who did not have postoperative delirium served as the non-delirium control group (197 patients, one case of a 65 year old man was excluded because he was transported to another hospital upon the request of his relatives on the second day after the operation).

\section{Preoperative factors}

The mean age of delirium group and non-delirium group were 68.9 and 63.7 years old $(P=0.004)$, and the difference was also significant for the percentage of patients older than 65 years $(P=0.003)$. Delirium subjects were treated with more medications preoperatively than controls (mean 3.7 vs. 2.4 , respectively, $P=0.000$ ), and there were more patients with comorbid diabetes mellitus $(P=0.042)$ and central nervous system disorders $(P=0.013)$ in delirium group compared with the control group. Nine patients in delirium group and 46 in non-delirium group had previously undergone operations $(P=0.028)$. No significant differences were found for the other factors (Tables 1,2).

\section{Intraoperative factors}

Eight patients with delirium and 37 patients without delirium were transfused with $800 \mathrm{~mL}$ or more blood
$(P=0.024)$. There were no differences for the other factors (Tables 1, 2).

Postoperative factors

The sodium level on the first day after the operation in delirium group was lower than what was observed in the control group (139.5 vs. $141, P=0.029$ ) and the blood sugar level was higher in the delirium group compared with the control group (7.9 vs. 6.9, $P=0.027$ ). No differences were found between groups for the other factors (Tables 1,2).

\section{Logistic regression analysis}

Variables such as age $>65$ years, treatment with multiple drugs ( $>$ three kinds) on the previous days, audition impairments, diabetes mellitus, central nervous system disorder, pulmonary disease, surgical history, blood loss $\geq 800 \mathrm{~mL}$, blood transfusion $\geq 800 \mathrm{~mL}$ and postoperative laboratory data including hemoglobin $<100 \mathrm{~g} / \mathrm{L}$, hematocrit $<30 \%$, sodium (dichotomized at $139 \mathrm{mmol} / \mathrm{L}$ ), blood sugar (dichotomized at $7 \mathrm{mmol} / \mathrm{L}$ ) were all included in logistic regression analysis according to results of the previous univariate analysis. The results showed that central nervous system disorder (OR 6.480), surgical history (OR 3.499), age $>65$ years (OR 3.390), diabetes mellitus (OR 2.981), blood transfusion $\geq 800 \mathrm{~mL}$ (OR 2.537), and hemoglobin $<100 \mathrm{~g} / \mathrm{L}$ (OR 0.281 ) were significantly related to the occurrence postoperative delirium (Table 3).

\section{Discussion}

Though postoperative delirium in other surgeries had been widely reported $[5,13,17,19,21-24]$, there is only one known article relative to postoperative delirium in spinal surgery, which was reported by Kawaguchi [12]. He speculated that there were few articles in this field because the incidence of postoperative delirium is lower in this patient population. In his research, the incidence of postoperative delirium was $3.8 \%$, which is close to our result and lower than hip fracture surgery. This may be because patients who have undergone spinal surgery are exposed to less stressor than patients with hip fracture; most patients with hip fracture go directly from the emergency room to surgery $[12,21]$.

Postoperative delirium can easily occur in older patients, and this condition has become widely accepted [2,9]. There were 13 patients older than 65 years who had postoperative delirium, and in logistic regression analysis this factor had a high OR ratio of 3.390. Brown reported approximately one-quarter of adults aged 65 and older experience delirium during hospitalization [3]. Ergina 
Table 1 Association between factors of categorical variables and postoperative delirium

a No significant difference

\begin{tabular}{|c|c|c|c|}
\hline & Delirium & Non-delirium & $P$ value \\
\hline \multicolumn{4}{|l|}{ Preoperative Data } \\
\hline Male & 10 & 103 & $-{ }^{\mathrm{a}}$ \\
\hline Age $>65$ years & 13 & 71 & 0.003 \\
\hline Solitary life & 3 & 15 & $-{ }^{\mathrm{a}}$ \\
\hline \multicolumn{4}{|l|}{ Drugs } \\
\hline Alcohol habit ( $\geq 30 \mathrm{~g} /$ day $)$ & 1 & 13 & $-{ }^{\mathrm{a}}$ \\
\hline Sedative-hypnotic drugs use & 11 & 128 & $-{ }^{\mathrm{a}}$ \\
\hline $\begin{array}{l}\text { Treatment with multiple drugs (>three kinds) } \\
\text { on the days prior to surgery }\end{array}$ & 7 & 34 & $-^{\mathrm{a}}$ \\
\hline Long preoperative hospital stay ( $>10$ days) & 3 & 17 & $-{ }^{\mathrm{a}}$ \\
\hline Malnutrition & 3 & 12 & $-{ }^{\mathrm{a}}$ \\
\hline \multicolumn{4}{|l|}{ Sensory impairments } \\
\hline Vision impairments & 1 & 4 & $-{ }^{\mathrm{a}}$ \\
\hline Audition impairments & 2 & 4 & $-{ }^{\mathrm{a}}$ \\
\hline \multicolumn{4}{|l|}{ Comorbid diseases } \\
\hline Hypertension & 4 & 58 & $-{ }^{\mathrm{a}}$ \\
\hline Diabetes mellitus & 6 & 25 & 0.042 \\
\hline Pulmonary disease & 2 & 7 & $-^{\mathrm{a}}$ \\
\hline Cardiac disease & 2 & 14 & $-{ }^{\mathrm{a}}$ \\
\hline Central nervous system disorder & 4 & 9 & 0.013 \\
\hline Psychiatric disorder & 1 & 1 & $-{ }^{\mathrm{a}}$ \\
\hline Chronic kidney disease & 1 & 6 & $-{ }^{\mathrm{a}}$ \\
\hline Chronic liver disease & 1 & 11 & $-{ }^{\mathrm{a}}$ \\
\hline Surgical history & 9 & 46 & 0.028 \\
\hline History of delirium & 1 & 0 & $-^{\mathrm{a}}$ \\
\hline \multicolumn{4}{|l|}{ Intraoperative Data } \\
\hline Operative time $\geq 180 \mathrm{~min}$ & 10 & 80 & $-{ }^{\mathrm{a}}$ \\
\hline Blood loss $\geq 800 \mathrm{~mL}$ & 9 & 68 & $-{ }^{\mathrm{a}}$ \\
\hline Blood transfusion $\geq 800 \mathrm{~mL}$ & 8 & 37 & 0.024 \\
\hline Total infusion $\geq 3,000 \mathrm{~mL}$ & 10 & 102 & $-{ }^{\mathrm{a}}$ \\
\hline Surgical implants used & 17 & 186 & $-{ }^{\mathrm{a}}$ \\
\hline \multicolumn{4}{|l|}{ Postoperative Data } \\
\hline Analgesic pumps & 18 & 188 & $--^{\mathrm{a}}$ \\
\hline Fever (temperature $>38^{\circ} \mathrm{C}$ ) & 0 & 4 & $-{ }^{\mathrm{a}}$ \\
\hline Hemoglobin $<100 \mathrm{~g} / \mathrm{L}$ & 7 & 37 & $-{ }^{\mathrm{a}}$ \\
\hline Hematocrit $<30 \%$ & 8 & 42 & $-^{\mathrm{a}}$ \\
\hline
\end{tabular}

suggested that this may be because older adults have disproportionably more operations than younger adults, and also because the elderly population is growing [7]. All of the patients in our study were under general anesthesia, and one potential candidate mechanism of cognitive impairment is general anesthesia itself [6]. General anesthesia affects brain function at all levels, including neuronal membranes, receptors, ion channels, neurotransmitters, cerebral blood flow, and metabolism [8]. Moreover, the aged brain is more susceptible to anesthetic effects and more sensitive to nonanesthetic drugs [16]. We also found that a greater percentage of patients in delirium group had a history of surgery than in the non-delirium group, with an OR ratio of 3.499 in logistic regression analysis. This finding is in accord with Ergina's research.

In Amador's review, he thought one of the greatest predisposing risk factors for developing postoperative delirium was the presence of neurodegenerative diseases such as Alzheimer's or Parkinson's disease [2]. The same was found in our analysis; central nervous system disorder had the highest OR ratio of 6.480 in logistic regression analysis. Four patients in delirium group had comorbid central nervous system disorders (vs. 9 in the control group, $P=0.013)$. Of the four patients, three had 
Table 2 Association between factors of numerical variables/ ordinal variables and postoperative delirium

\begin{tabular}{|c|c|c|c|}
\hline & Delirium & Non-delirium & $P$ value \\
\hline \multicolumn{4}{|l|}{ Preoperative Data } \\
\hline Age (years) & $68.9 \pm 8.4$ & $63.7 \pm 7.3$ & 0.004 \\
\hline Number of medications & $3.7 \pm 1.4$ & $2.4 \pm 1.1$ & 0.000 \\
\hline Preoperative hospital days & $7.6 \pm 3.9$ & $6.7 \pm 3.4$ & - \\
\hline Nutrition status (total protein, g/L) & $66.1 \pm 8.3$ & $67.7 \pm 5.9$ & - \\
\hline Number of comorbid diseases & $1.8 \pm 2.0$ & $1.2 \pm 1.1$ & - \\
\hline Blood pressure (SBP, mmHg) & $128 \pm 16.4$ & $130 \pm 15.7$ & - \\
\hline Hemoglobin (g/L) & $132 \pm 14.3$ & $134 \pm 14.8$ & - \\
\hline Hematocrit $(\%)$ & $39.5 \pm 4.3$ & $40.4 \pm 4.1$ & - \\
\hline \multicolumn{4}{|l|}{ Blood biochemistry } \\
\hline Sodium (Na) & $141.0 \pm 3.2$ & $142.0 \pm 2.8$ & - \\
\hline Potassium (K) & $4.1 \pm 0.4$ & $4.0 \pm 0.3$ & - \\
\hline Blood sugar (mmol/L) & $6.0 \pm 1.5$ & $5.5 \pm 3.0$ & - \\
\hline \multicolumn{4}{|l|}{ Intraoperative Data } \\
\hline Operative time (min) & $182.8 \pm 66.5$ & $167.1 \pm 68.1$ & - \\
\hline Blood loss (mL) & $937.2 \pm 795.1$ & $716.9 \pm 632.4$ & - \\
\hline Blood transfusion $(\mathrm{mL})$ & $656.6 \pm 796.5$ & $376.9 \pm 640.5$ & - \\
\hline Total infusion $(\mathrm{mL})$ & $3,227.8 \pm 1,220.8$ & $3,046.2 \pm 1,242.5$ & - \\
\hline \multicolumn{4}{|l|}{ Postoperative Data } \\
\hline Blood pressure (SBP, mmHg) & $129.1 \pm 15.5$ & $125.8 \pm 15.1$ & - \\
\hline Hemoglobin $(\mathrm{g} / \mathrm{L})$ & $106.4 \pm 15.0$ & $112.7 \pm 16.8$ & - \\
\hline Hematocrit $(\%)$ & $31.3 \pm 4.2$ & $33.4 \pm 3.4$ & - \\
\hline \multicolumn{4}{|l|}{ Blood biochemistry } \\
\hline Sodium (mmol/L) & $139.5 \pm 2.5$ & $141.0 \pm 2.82$ & 0.029 \\
\hline Potassium (mmol/L) & $3.94 \pm 0.5$ & $3.80 \pm 0.4$ & - \\
\hline Blood sugar $(\mathrm{mmol} / \mathrm{L})$ & $7.9 \pm 2.0$ & $6.9 \pm 1.8$ & 0.027 \\
\hline
\end{tabular}

Table 3 Results of logistic regression analysis

\begin{tabular}{lllllc}
\hline Factors & B & Sb & Wald $x^{2}$ & $P$ value & $\begin{array}{l}\text { Odds Ratio } \\
(95 \% \text { confidence interval) }\end{array}$ \\
\hline Central nervous system disorder & 1.869 & 0.787 & 5.644 & 0.018 & $6.480(1.387-30.278)$ \\
Surgical history & 1.253 & 0.562 & 4.959 & 0.026 & $3.499(1.162-10.538)$ \\
Age $>65$ years & 1.221 & 0.587 & 4.323 & 0.038 & $3.390(1.073-10.717)$ \\
Diabetes mellitus & 1.092 & 0.604 & 3.267 & 0.071 & $2.981(0.912-9.746)$ \\
Blood transfusion $\geq 800 \mathrm{~mL}$ & 0.931 & 0.577 & 2.605 & 0.107 & $2.537(0.819-7.856)$ \\
Hemoglobin $<100 \mathrm{~g} / \mathrm{L}$ & -1.268 & 0.608 & 4.354 & 0.037 & $0.281(0.086-0.926)$ \\
\hline
\end{tabular}

Age $>65$ years, treatment with multiple drugs ( $>$ three) on the days prior to surgery, audition impairments, diabetes mellitus, central nervous system disorder, pulmonary disease, surgical history, blood loss $\geq 800 \mathrm{~mL}$, blood transfusion $\geq 800 \mathrm{~mL}$, postoperative hemoglobin $<100 \mathrm{~g} / \mathrm{L}$, hematocrit $<30 \%$, sodium (dichotomized at $139 \mathrm{mmol} / \mathrm{L}$ ), and blood sugar (dichotomized at $7 \mathrm{mmol} / \mathrm{L}$ ) were all included in logistic regression analysis

Parkinson's disease (none has the Parkinson's disease in control group), and none had Alzheimer's disease in our study.

Drug toxicity, due to drugs such as meperidine [1], accounts for approximately $30 \%$ of all cases of delirium and plays a major role in postoperative delirium [2]. There were no patients treated with meperidine before or after operation in our study. In other reports, alcohol abuse is an important risk factor $[1,2,4,5,18]$; however, this does not seem to be the case in our research, possibly because few Chinese people have alcohol problems compared with Western populations.

Operative time more than $10 \mathrm{~h}$, blood transfusion more than $800 \mathrm{~mL}$, and infusion more than $5,000 \mathrm{~mL}$ were considered significant risk factors in Yamagata's research [23]. However, there were no generally accepted standards or ranges of values for these factors because different surgeries had different features. Similar results can also be 
found in the HCT, and sodium levels on the first day after surgery for the delirium group, which were lower than those in the control group in Kawaguchi's research [12]. The present study also demonstrated that the lower concentrations of HGB and HCT on the first day after surgery had close relation to postoperative delirium [12, 17]. Kawaguchi's group stated that the patients with the HCT $<30 \%$ were at risk for postoperative delirium. A low concentration of HGB and HCT might reduce the oxygen supply to the brain and bring about postoperative delirium. The difference of sodium levels between the delirium and control patients on the first day after surgery has only been presented in these two spinal research reports. Although there was a difference, the sodium levels in both reports were in normal range, so it may have no value in the incidence of postoperative delirium. Morphine used for postoperative pain relief had been related to postoperative delirium [21], and there were no patients treated with morphine in our study. Rather, all were treated with fentanyl, which does not appear to increase the risk for delirium.

The risk for delirium is multifactorial $[10,11]$. There still are many factors that we did not assess, such as preoperative mental status, low postoperative oxygen saturation, and pain. Many prospective studies used the Mini-Mental Status Exam [MMSE] for the patients to evaluate the mental status, Lynch et al. [15] found that higher pain scores at rest during the first three postoperative days were associated with an increased risk of delirium. In a retrospective study such as the one that we conducted, data like this cannot be collected. Although we did not evaluate these factors, further prospective studies should be carried out to investigate the relationship between postoperative delirium and these factors in detail.

In conclusion, this study confirms previous findings and suggests that there is an incidence of postoperative delirium in spinal surgery. Older age, comorbid diseases like diabetes mellitus or central nervous system disorder, surgical history, blood transfusion $\geq 800 \mathrm{~mL}$, and low concentrations of HCT/HGB on the first day after surgery are probable risk factors for postoperative delirium in spinal surgery. Understanding the risk factors of delirium can improve its prevention, diagnosis, and management. Risk of postoperative delirium can be reduced with careful attention to risk factors [2]. However, more prospective research is necessary in order to evaluate factors in greater detail.

\section{References}

1. Adunsky A, Levy R, Heim M et al (2002) Meperidine analgesia and delirium in aged hip fracture patients. Arch Gerontol Geriatr 35:253-259. doi:10.1016/S0167-4943(02)00045-6
2. Amador LF, Goodwin JS (2005) Postoperative delirium in the older patient. J Am Coll Surg 200:767-773. doi:10.1016/j. jamcollsurg.2004.08.031

3. American Psychiatric Association (1994) Diagnostic and statistical manual of mental disorders, 4th edn. American Psychiatric Association, Washington DC

4. Brown TM, Boyle MF (2002) Delirium. BMJ 325:644-647. doi: 10.1136/bmj.325.7365.644

5. Bucerius J, Gummert JF, Borger MA et al (2004) Predictors of delirium after cardiac surgery delirium: effects of beating-heart (off-pump) surgery. J Thorac Cardiovasc Surg 127:57-64. doi: 10.1016/S0022-5223(03)01281-9

6. Culley DJ, Baxter M, Yukhananov R et al (2003) The memory effects of general anesthesia persist for weeks in young and aged rats. Anesth Analg 96:1004-1009. doi:10.1213/01.ANE. 0000052712.67573 .12

7. Ergina PL, Gold SL, Meakins JL (1993) Perioperative care of the elderly patient. World J Surg 17:192-198. doi:10.1007/ BF01658926

8. Franks NP, Lieb WR (1994) Molecular and cellular mechanisms of general anaesthesia. Nature 367:607-614. doi:10.1038/ $367607 \mathrm{a} 0$

9. Inouye SK (2006) Delirium in older persons. N Engl J Med 354:1157-1165. doi:10.1056/NEJMra052321

10. Inouye SK, Charpentier PA (1996) Precipitating factors for delirium in hospitalized elderly persons: predictive model and interrelationship with baseline vulnerability. JAMA 275:852857. doi:10.1001/jama.275.11.852

11. Inouye SK, Viscoli CM, Horwitz RI et al (1993) A predictive model for delirium in hospitalized elderly medical patients based on admission characteristics. Ann Intern Med 119:474-481

12. Kawaguchi Y, Kanamori M, Ishihara H et al (2006) Postoperative delirium in spine surgery. Spine J 6:164-169. doi:10.1016/ j.spinee.2005.06.010

13. Kazmierski J, Kowman M, Banach M et al (2006) Preoperative predictors of delirium after cardiac surgery: a preliminary study. Gen Hosp Psychiatry 28:536-538. doi:10.1016/ j.genhosppsych.2006.08.007

14. Koolhoven I, Tjon-A-Tsien MR, van der Mast RC (1996) Early diagnosis of delirium after cardiac surgery. Gen Hosp Psychiatry 18:448-451. doi:10.1016/S0163-8343(96)00089-8

15. Lynch EP, Lazor MA, Gellis JE et al (1998) The impact of postoperative pain on the development of postoperative delirium. Anesth Analg 86:781-785. doi:10.1097/00000539-199804000-00019

16. Magnusson KR, Scanga C, Wagner AE et al (2000) Changes in anesthetic sensitivity and glutamate receptors in the aging canine brain. J Gerontol A Biol Sci Med Sci 55:B448-B454

17. Marcantonio ER, Goldman L, Orav EJ et al (1998) The association of intraoperative factors with the development of postoperative delirium. Am J Med 105:380-384. doi:10.1016/ S0002-9343(98)00292-7

18. O'Brien D (2002) Acute postoperative delirium: definitions, incidence, recognition, and interventions. J Perianesth Nurs 17:384-392. doi:10.1053/jpan.2002.36783

19. Schneider F, Bohner H, Habel U et al (2002) Risk factors for postoperative delirium in vascular surgery. Gen Hosp Psychiatry 249:28-34. doi:10.1016/S0163-8343(01)00168-2

20. Schuurmans MJ, Duursma SA, Shortridge-Baggett LM (2001) Early recognition of delirium: review of the literature. J Clin Nurs 10:721-729. doi:10.1046/j.1365-2702.2001.00548.x

21. Schuurmans MJ, Duursma SA, Shortridge-Baggett LM et al (2003) Elderly patients with a hip fracture: the risk for delirium. Appl Nurs Res 16:75-84. doi:10.1016/S0897-1897(03)00012-0

22. William-Russo P, Urquhart BL, Sharrock NE et al (1992) Postoperative delirium: predictors and prognosis in elderly orthopedic patients. J Am Geriatr Soc 40:759-767 
23. Yamagata K, Onizawa K, Yusa $\mathrm{H}$ et al (2005) Risk factors for postoperative delirium in patients undergoing head and neck cancer surgery. Int J Oral Maxillofac Surg 34:33-36. doi: 10.1016/j.ijom.2004.03.005
24. Zakriya KJ, Christmas C, Wenz JF Sr et al (2002) Preoperative factors associated with postoperative change in confusion assessment method score in hip fracture patients. Anesth Analg 94:1628-1632. doi:10.1097/00000539-200206000-00050 